\title{
NODE.Health Meeting Report and Panel Discussion - The FDA's Changing Regulatory Landscape for Digital Health Technologies and Digital Health Innovation during COVID-19: A Discussion with Eric Topol and Bakul Patel, Moderated by Aenor Sawyer
}

\author{
Anna Andoni ${ }^{a}$ Shayann Ramedani ${ }^{b}$ Benjamin I. Rosner ${ }^{c}$ Aenor Sawyer ${ }^{d}$ \\ ${ }^{a}$ NODE. Health, New York, NY, USA; b Penn State Clinical and Translational Science Institute, \\ University Park, PA, USA; ' Division of Hospital Medicine, University of California, San \\ Francisco, CA, USA; ${ }^{d}$ Translational Research Institute for Space Health, San Francisco, CA, \\ USA
}

Keywords

Digital biomarkers $\cdot$ Evidence $\cdot$ Regulatory landscape $\cdot$ COVID-19 $\cdot$ Wearables

\section{Introduction}

On August 19th, 2020, the Network of Digital Evidence, NODE.Health, a 501(c)3 nonprofit organization dedicated to accelerating the adoption of digital health technology by creating and promoting best practices in evidence and transformation, hosted a webinar about the changing FDA digital health regulatory landscape and the need to balance speed with evidence [1]. The session included guest panelists Dr. Eric Topol, Founder and Director of the Scripps Research Translational Institute, and Bakul Patel, Director of Digital Health at the Center for Devices and Radiological Health at the Food and Drug Administration (FDA).

The NODE.Health Webinars are part of a series of presentations, discussions, sessions, and other events open to the public and to NODE.Health's membership that are intended to bring expert perspectives on topics related to evidence, digital health, and innovation. These sessions continue during a dedicated week as part of NODE.Health's Digital Medicine Conference scheduled to be held from December 7th to December 11th, 2020. While NODE.Health supports the causes of many of its speakers, NODE. Health does not necessarily endorse any product or belief mentioned in these presentations or beyond. Elements of the webinar have been redacted or summarized for clarity. 
Dr. Topol is a prominent researcher with over 1,200 peer-reviewed articles, is one of the world's experts in evidence-based digital health, and is the author of "The Patient Will See You Now." Mr. Patel focuses on the regulatory landscape of digital health technologies and innovating new processes of regulation. Mr. Patel has been a leading proponent of the now ubiquitous term Software as Medical Device (SaMD), and is currently leading the FDA's development of the Digital Health Software Pre-Certification Program to reimagine a regulatory framework for digital health tools that balances the acceleration of evaluation and approval of such tools with scientific rigor. The session was moderated by Dr. Aenor Sawyer, board member of NODE.Health, orthopedist at the University of California San Francisco (UCSF), and Chief Health Information Officer (CHIO) at the NASA funded Translational Research Institute for Space Health with over a decade of experience in development and evaluation of device, digital health, telehealth, and electronic health record solutions. The webinar began with Dr. Sawyer introducing Dr. Topol and Mr. Patel who then delivered individual presentations. A moderated discussion about digital health and the regulatory landscape ensued.

\section{Webinar Content}

Mr. Patel gave the first presentation, focusing on how the FDA aims to iteratively tailor its digital health regulatory oversight to advances in digital health technologies, and to build an evolving evidence infrastructure. Mr. Patel shared that the FDA defines digital health technologies as the convergence of computing power, connectivity, sensors and software used in healthcare. These digital health technologies can be used as medical products, incorporated into medical products (including pharmacologic products), used to develop medical products, used to study medical products, or used as adjuncts or companions to medical products including diagnostics and therapeutics. He also shared that the current coronavirus pandemic has accentuated some of the questions around how the FDA evaluates and/or accelerates various digital health products to market.

Next, Mr. Patel highlighted the need to create a mechanism and infrastructure for people to learn what is happening in real clinical practice by bringing to the surface evidence from digital health technologies. He explained that in its mission, the FDA intends to move from episodic oversight to continuous oversight that creates trust by using a pragmatic check-in with organizations generating real-world performance data. Mr. Patel described the FDA's SaMD risk framework, the same framework adopted by the International Medical Device Regulators Forum, using two dimensions that are applicable today: the state of the patient's healthcare situation (namely, how serious is the patient's condition in which the SaMD will be used?) and the significance of the information provided by the SaMD to the healthcare decision under consideration (namely, what is it doing? Does it merely inform clinical management, drive clinical management, or does it treat or diagnose?). The safety and efficacy of digital health solutions become clearer in the context of such risk frameworks.

Mr. Patel also highlighted that it is important to build evidence incrementally, indicating that in digital health, not everything may need to go through prospective studies, but that as technology iterates we need to also create the mechanisms, infrastructure, and avenues for people to learn what's happening in real practice and bring back these learnings to the digital health solution developers, as happens normally in other engineering and business practices. He encouraged SaMD manufacturers to leverage SaMD technology capabilities to capture real-world performance data, to understand user interactions with SaMD, and to conduct ongoing monitoring of analytical and technical performance to support future intended uses.

In order to provide a framework for iterative solutions, Mr. Patel demonstrated the FDA's Real World Performance Analytics Framework, intended to tailor the regulatory approach to

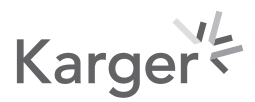


fit the evolving digital health space. The framework shows that product performance, user experience, and health analytics can be brought together and used to evaluate and better understand what is happening with SaMD products.

Mr. Patel concluded his presentation by stating that the regulatory field is in transition. In this transitional period, emerging regulatory challenges can be addressed by supplementing the go-to-market phased-gate regulatory approach with continuous oversight involving a total product life cycle (how the products are used, who is making them, and what the product is). He stated that the FDA is undertaking continuous learning cycles, and that it is important to keep the regulatory system nimble in order to accommodate digital health tools and real-world evidence. As it evolves, Mr. Patel indicated that the FDA will be considering new measures, accommodating new trials outside of traditional settings, examining patient generated and reported data, and acknowledging that the gold standards will change.

Following Mr. Patel's presentation, Dr. Topol introduced the current state of digital technologies and their potential use cases during the COVID-19 pandemic. He exemplified this by indicating that polymerase chain reaction (PCR) testing for coronavirus ought to and can be supplemented with real-time digital technologies. In his recent paper, he and other investigators used a smartwatch application in tens of thousands of people to capture changes in heart rate and fatigue (as determined by sleep and step count patterns) to serve as a proxy to detect clusters of influenza-like illness in real-time, and predict outbreaks... With over 100 million people owning smartwatches, the potential to disseminate and adopt this form of surveillance is broad. Unfortunately, per Dr. Topol, the delay in FDA approval of rapid tests for coronavirus has left the country with little means to determine in real-time when a person is infectious, as opposed to being infected. Given the limitations associated with turnaround times on the order of days to weeks for PCR tests, Dr. Topol indicated that we should be embracing digital surveillance in this vacuum, stating that digital surveillance, because of its real-time performance is our pandemic exit strategy. Finally, Dr. Topol discussed that in Germany, the Robert Koch Institute developed an app to track resting heart rate and steps as proxies for COVID-19, with their tool gaining adoption by hundreds of thousands of users within hours of deployment because unlike in the U.S., where the Centers for Diseases Control has been suppressed in recent months, there is trust in the national disease surveillance infrastructure. The other problem he identified is that unlike vaccines and drugs, "Digital is like Rodney Dangerfield. We don't get no respect."

After the individual presentations about the current regulatory environment for digital health technologies and the potential use cases for digital health technologies during public health crises such as the COVID-19 pandemic, a panel discussion moderated by Dr. Sawyer followed that identified the potential for digital biomarkers during the pandemic in identifying disease clusters. Mr. Bakul agreed that evidence from digital health tools has been considered second class to date, indicating that current gold standards need to be upgraded in order to provide better evidence for reimbursement and authorizations for digital health tools. Mr. Patel also emphasized the need to have data interoperability and centralized data collation in order to be able to see signals emerge, and to engender acceptance in the community, as has been accomplished in Germany. He stated that the concept of triangulation, or looking at multiple sources of signals or different data streams to gain better diagnostic accuracy needs to be more broadly applied to digital medicine in a manner analogous to how the telecommunications industry uses triangulation to identify geolocation.

Dr. Topol followed, describing that we do not know a lot about curbing transmission from asymptomatic individuals because the $30-40 \%$ of people who are asymptomatic rarely get tested, even though they may be highly infectious. However, if digital biomarkers were to be used even in asymptomatic patients such as through smartwatches, we can likely detect infectiousness pre-symptomatically. Navigating out of the pandemic with digital biomarkers, however, will take concerted federal initiative.

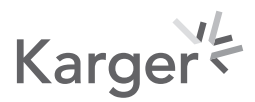


With respect to digital tools, Mr. Patel indicated that the FDA remains on a journey to understand total product lifecycle and the uncertainties of digital signals before digital tools go to market. But Mr. Patel stated that the FDA is not there yet with a fully agile precertification program that can tackle challenges such as those brought on by the pandemic. The question was raised by Dr. Sawyer as to whether regulation and standardization on digital wearable manufacturers might therefore raise the quality and acceptability of digital biomarkers. Mr. Patel described the complexities of this, giving examples in which companies use proprietary algorithms to process the raw biomarkers, and these algorithms are not standardized. In order to facilitate opportunities for consumer wearables and digital health technologies to be used in healthcare, the FDA is collaborating with organizations such as the Consumer Technology Association (CTA) to come up with necessary standardization and transparency to drive the acceptance of these digital health products. Dr. Sawyer suggested that centers such as the Scripps Research Translational Institute, who are doing innovative validation work might inform the FDA on their evaluation and approval processes with novel methodologies, a suggestion to which Dr. Topol and Mr. Patel concurred. Dr. Topol further indicated that additional emerging wearable biomarker signals such as those from pulse oximetry might provide valuable data about disease, but that such technologies have largely remained proprietary, and unvalidated in persons with different skin pigmentation.

Dr. Aenor asked if it would be possible to accelerate innovation and still bring digital health tools to market with scientific rigor. Mr. Patel encouraged manufacturers of digital health technology developers to start thinking about instrumentation of products for positive benefits and to capture the variables from these products as evidence while in use which may, in some ways, be superior to doing a randomized controlled trial (RCT). For example, one can argue that real-world evidence, or evidence while in use, is better than trying to do an RCT since RCT's are limited in their breadth of participants compared to observational or postmarket studies. Dr. Topol concurred that RCTs are contrived because inclusion and exclusion criteria render them selective and not necessarily representative of the real world. Ultimately, both Topol and Patel believed that it takes a combination of real-world use, different study approaches, and data gathering that can improve evidence and enable accelerated market approval of digital health technologies.

Dr. Sawyer asked the panelists about windows of data gathering under Emergency Use Authorization (EUA) for products where the risk may be low. Mr. Patel encouraged manufacturers to take advantage of this unique EUA opportunity that is available in the current pandemic health crisis. According to Eric Topol, the EUA has been used as an accelerator to put out new modalities of testing and health technologies. However, an example of a side effect of this during the pandemic was the marketing of useless or shoddy tests, resulting in misleading results where there were not enough data to support their use. Dr. Topol stated that there needs to be a conditional approval mechanism in which digital health products may be accelerated to market conditionally but must report results back for ongoing market approval. According to Mr. Parel, this type of follow-up has been challenging, as the FDA has been more focused on adverse events and not factoring in the positive benefits of prospective or post-market studies. However, it was felt that we should consider such types of approaches through a different mechanism for low-risk devices such as digital health technologies in order to build on their evidence.

Finally, Dr. Sawyer fielded questions from the audience. The first question to Mr. Patel was "What does the FDA consider its role in neutralizing the digital divide and disparities?" Mr. Patel indicated that access to connectivity, or access to devices themselves are different dimensions of the digital divide. He stated that the FDA is deeply interested in improving access to devices, but could not answer for connectivity issues such as those managed by the 
Federal Communications Commission. Dr. Topol responded by stating that there are many communities with limited data plans and limited access to devices, but that it would be smart as a nation, if we supplied these to everyone. However, with 100 million Americans who own fitness trackers, he suggested that if we were to encourage everyone who owns one to use it or to make it available to those who do not have such devices, we could achieve a lot more in digital pandemic surveillance. A final question from the audience was, "Why should digital health get the same attention as vaccines?" Dr. Topol responded by saying that everyone reacts differently to vaccines and there will be some untoward effects of vaccines on some people. We have invested enormously in vaccines, and they are expensive to make. But he counters with the question, "What is the risk of digital?" The worst we can do, he says, is generate a false positive such as thinking we are detecting COVID when in fact it is the flu. But, he added, that we are not creating sickness with digital as vaccines will do to some. Digital is much safer, can be done at scale, and is cheap compared to vaccines. Interestingly, some vaccine companies have supplemented their vaccine work with digital tools for tracking, a unique opportunity Dr. Topol had not previously considered.

In wrapping up, Mr. Patel stated that in 2020, despite all of our computing power, we still have not modeled human behavior, but he hopes we can accelerate that. Dr. Topol stated that we have a clinical trial impaction syndrome in the US and need to accelerate. Dr. Topol highly encouraged rapid test development for coronavirus, indicating that such tests must be developed quickly, and paid for by the government.

\section{Conclusion}

FDA regulation of digital health technologies is an evolving and iterative process. The pandemic has given the fastest route to deployment of digital health technologies through EUAs; however, there is still a long way to go in creating agile, real-world evidence-based regulatory pathways that reflect the desire to accelerate digital health tools to market, and the needs for evidence-based evaluation of these tools for safety and efficacy. Updated requirements and new gold standards for digital health validation as well as standardization are needed to drive new regulatory pathways that are beneficial to the field, safe, and effective for the end-users on both an individual and population level.

\section{Conflict of Interest Statement}

Dr. Aenor Sawyer is on the NODE.Health Executive Board. Dr. Benjamin Rosner declares equity interest in Infermedica and serves as NODE.Health's Scientific Advisor. Dr. Anna Andoni is NODE.Health's Program Manager, and Shayann Ramedani is a NODE.Health Scholar.

\section{Funding Sources}

NODE.Health is a 501(c)3 that receives sponsorship funding from technology companies in support of the NODE.Health Mission. Sponsors were highlighted at the end of the webinar in appreciation for their support. 


\section{Author Contributions}

Aenor Sawyer served as moderator for the NODE.Health Webinar and senior author to this transcript. Anna Andoni and Shayann Ramedani translated the webinar into this transcript. Benjamin Rosner provided oversight and proof of the manuscript, as well as advisory support as lead of the NODE.Health Scholar Team.

\section{References}

1 NODE.Health Website. The Changing Regulatory Landscape during and after COVID-19 featuring Dr. Eric Topol, Bakul Patel and moderated by Dr. Aenor Sawyer [August 19th, 2020]. Available from: https://nodehealth.org/2020/09/16/the-changing-regulatory-landscape-during-and-after-covid-19/ 